\title{
Processing of telecommunication signals using periodically poled lithium niobate waveguides
}

\author{
Periklis Petropoulos ${ }^{1}$, Sheng Liu ${ }^{1}$, Kwang Jo Lee ${ }^{1}$, Francesca Parmigiani ${ }^{1}$, \\ Katia Gallo ${ }^{2}$ and David J. Richardson ${ }^{1}$ \\ ${ }^{1}$ Optoelectronics Research Centre, University of Southampton, Southampton SO17 1BJ, \\ United Kingdom \\ ${ }^{2}$ Department of Applied Physics, Royal Institute of Technology (KTH), 10691 Stockholm, Sweden
}

\begin{abstract}
This talk reports on processing of high-speed telecommunication signals based on cascaded quadratic nonlinearities in periodically poled lithium niobate (PPLN) waveguides. A strong pump positioned within the acceptance bandwidth of the PPLN device can interact via a cascaded nonlinear process with a second signal, thereby facilitating a switch with a broad optical bandwidth and yielding an output located within the same wavelength band as the inputs. The combination of the PPLN switch with custom-designed optical filtering for pre-conditioning of the signals gives rise to a versatile pulse processing system exhibiting important advantages in terms of compactness and environmental stability [1, 2]. As an example, Fig.1 describes schematically how an optical time-division multiplexed (OTDM) signal can be converted through cascaded second-harmonic generation and difference-frequency generation to a wavelength-division multiplexed (WDM) signal. The experiments that have led to this demonstration will be described in the talk [2].
\end{abstract}

Another exciting possibility offered by the action of periodically poled waveguides relates to the coherent manipulation of the optical phase of signals. Recent demonstrations relating to phase-sensitive amplification [3], as well as novel applications effecting to the elimination of chirp from optical signals will also be outlined in the talk.

a)
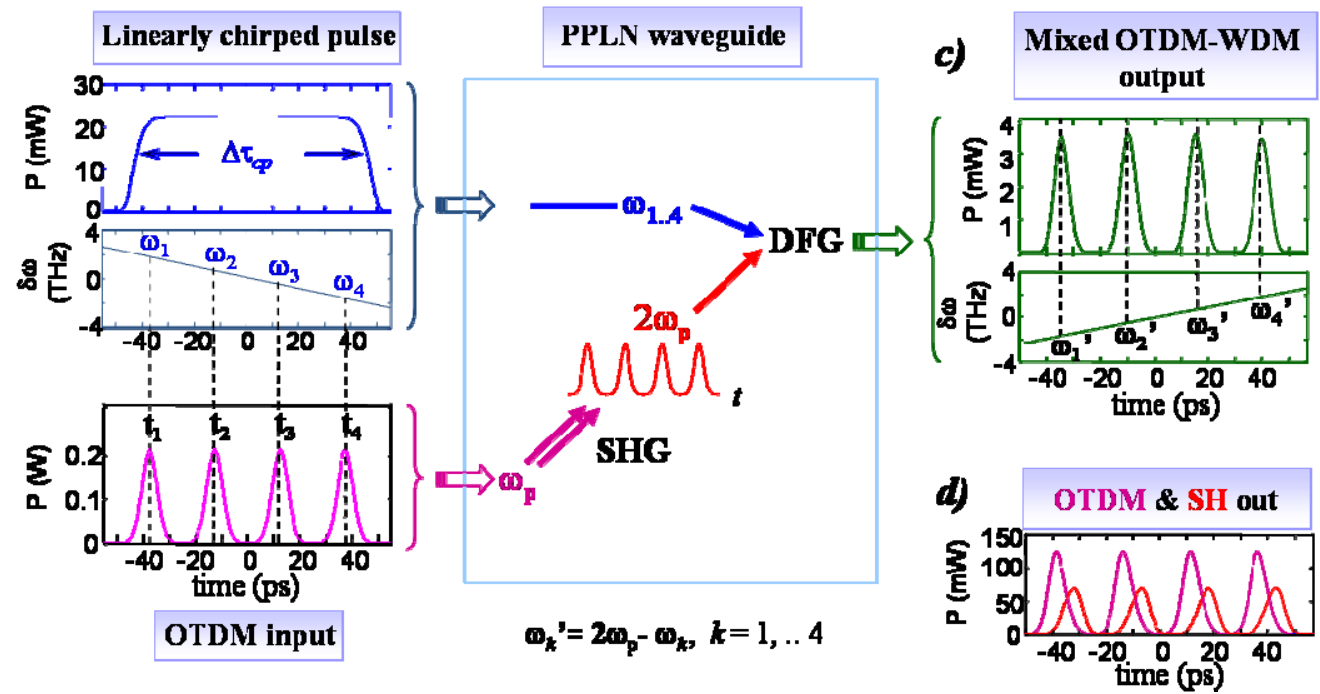

Fig.1 Schematic diagram illustrating the principle of OTDM to WDM conversion in a PPLN waveguide. 


\section{References}

[1] K. J. Lee, S. Liu, F. Parmigiani, P. Petropoulos, D. J. Richardson, and K. Gallo, "All-Optical Pulse Retiming Based on Quadratic Cascading in a Periodically Poled Lithium Niobate Waveguide " in Nonlinear Photonics, Karlsruhe, Germany, 2010, p. NMB5.

[2] K. J. Lee, S. Liu, F. Parmigiani, M. Ibsen, P. Petropoulos, K. Gallo, and D. J. Richardson, "OTDM to WDM format conversion based on quadratic cascading in a periodically poled lithium niobate waveguide," Opt. Express, vol. 18, pp. 10282-8, 2010.

[3] K. J. Lee, F. Parmigiani, S. Liu, J. Kakande, P. Petropoulos, K. Gallo, and D. J. Richardson, "Phase sensitive amplification based on quadratic cascading in a periodically poled lithium niobate waveguide," Optics Express, vol. 17, pp. 20393-400, 2009. 